\title{
ADAPTABILIDAD DE HÍBRIDOS TRIPLES DE MAÍZ Y DE SUS PROGENITORES PARA LA REGIÓN TROPICAL DEL SURESTE DE MÉXICO1
}

\author{
Mauro Sierra Macías ${ }^{2}$, Artemio Palafox Caballero ${ }^{2}$, Alejandro Espinosa Calderón ${ }^{3}$, Filiberto Caballero \\ Hernández ${ }^{4}$, Flavio Rodríguez Montalvo ${ }^{2}$, Sabel Barrón Freyre ${ }^{5}$, Roberto Valdivia Bernal ${ }^{6}$
}

\begin{abstract}
RESUMEN
Adaptabilidad de híbridos triples de maíz y de sus progenitores para la región tropical del sureste de México. Durante los ciclos primavera-verano de 1998 y 1999 y otoñoinvierno de 1997/98 y 1998/99, se condujo una investigación en los campos experimentales del INIFAP, Cotaxtla e Isla, Veracruz, Huimanguillo, Tabasco, y Apatzingán, Michoacan, para conocer el rendimiento, adaptabilidad y las características agronómicas de híbridos triples de maíz y sus progenitores. Se establecieron siete experimentos bajo diseños alfa-látice con dos repeticiones, en parcelas de un surco de $5 \mathrm{~m}$ de largo separados a $80 \mathrm{~cm}$, con una densidad de 62.500 plantas por hectárea. Con los híbridos sobresalientes se establecieron parcelas de validación durante los años 1999, 2002 y 2003, en los estados de Veracruz y Tabasco. Hubo híbridos triples estables, que superan en rendimiento e igualaron en características agronómicas al testigo comercial H-513. Dentro de los sobresalientes se encuentran: (1) H-513 x CABG3'- 10-1-6-1-1-1, el cual ha sido propuesto para su liberación oficial como H-520, (2) (CML247 x CML254) x CABG3'-3-4-2-1-3-1, (3) (LRB14 x D539) x CML254, H-513 x LT157, propuesto para su liberación como H-518 y la cruza. En la etapa de validación, los híbridos H-518 y H-520 registraron rendimientos similares al híbrido de cruza simple H-513, usado como progenitor hembra en la formación de estos híbridos. Se concluye que la formación de híbridos triples estables y de buen rendimiento, representa una alternativa de aprovechamiento de la heterosis con las ventajas adicionales en la producción de semillas.
\end{abstract}

Palabras clave: híbridos de maíz, interacción genético ambiental, heterosis, adaptabilidad.

\begin{abstract}
Adaptability of three-way hybrids of corn and their parental lines to the tropical region of South-Eastern México. During the spring-summer season 1998 and 1999 and fall-winter season of 1997/98 and 1998/99, a research about the adaptability of three-way hybrids of corn and their parents was carried at the Cotaxtla and Isla, Veracruz, Huimanguillo, Tabasco and Apatzingán, Michoacan experimental stations of INIFAP, located in the humid tropic of southeast of México. The objectives of this research were to assess yield, adaptability and agronomic characteristics of the three-way hybrids and their parents. Thus, seven experiments were carried out under an alpha lattice designs with two replications in plots of one 5 $\mathrm{m}$ long, $80 \mathrm{~cm}$ wide row and 62,500 plants per hectare. The best hybrids were established in validation with farmers during 1999, 2002 and 2003 in Veracruz and Tabasco states. Threeway hybrids were characterized as stable, upper or similar in yield than the control H-513. The best hybrids were: $\mathrm{H}-513 \mathrm{x}$ CABG3'-10-1-6-1-1-1, which has been suggested for official registration as H-520, (CML247 x CML254) x CABG3'-3-4-21-3-1, (LRB14 x D539) x CML254, H-513 x LT157, suggested for official registration as $\mathrm{H}-518$. In validation steps, we found that H-518 and H-520 got similar grain yield to H-513, wich participated as female parent in both three-way hybrids. In conclusion, we can say that getting three-way hybrids with good yield and agronomic characteristics represents an alternative to take advantage of heterosis with the additional advantage of seed production.
\end{abstract}

Key words: corn, three-way hybrids, genotype environment interaction, heterosis, adaptability.

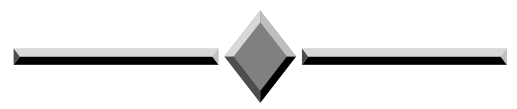

\footnotetext{
1 Recibido: 15 de setiembre, 2004. Aceptado: 17 de diciembre, 2004. Presentado en la L Reunión Anual del PCCMCA, El Salvador, 2004.

2 Campo Experimental Cotaxtla, INIFAP. Km 34 carr. Veracruz-Córdoba. Apdo. Postal 429. C.P. 91700, Veracruz, Ver. México. Correo electrónico: mauro_s55@hotmail.com

3 Campo Experimental Valle de México. Chapingo Texcoco estado de México. Correo electrónico: espinoale@yahoo.com.mx.

4 Campo Experimental Valle de Apatzingán. Michoacán, México. Correo electrónico: filicabah@ hotmail.com.

5 Campo Experimental Huimanguillo. Tabasco, México.

6 Campo Experimental Santiago Ixcuintla. Nayarit, México.
} 


\section{INTRODUCCIÓN}

En México se sembraron durante 2002, 6,48 millones de hectáreas de maíz para grano con un rendimiento medio de 2,32 t/ha y una producción de 13,02 millones de toneladas (SAGARPA 2002). En el sureste mexicano, se siembran anualmente 2,5 millones de hectáreas, de las cuales, cerca de un millón están comprendidas en las provincias agronómicas de buena y muy buena productividad y 100 mil hectáreas son sembradas bajo condiciones de riego. En esta superficie se recomienda preferentemente el uso de semilla de híbridos, los cuales expresan al máximo su potencial genético, dado por el efecto heterótico de cruzar progenitores de relativa divergencia genética (Reyes 1985; Gómez 1986; Vasal et al. 1992a; Vasal et al. 1992b). La adaptabilidad de los genotipos es importante porque permite conocer la respuesta a los diferentes ambientes definidos por el clima, el suelo y el manejo agronómico (Eberhart y Russell 1966). Los híbridos triples representan una alternativa interesante, ya que se aprovechan las ventajas que ofrece la heterosis en la producción comercial de maíz, al cruzar líneas de relativa divergencia genética con cruzas simples de alto rendimiento (Rodríguez et al. 1997). En la formación de híbridos es importante identificar líneas con buena aptitud combinatoria, buen rendimiento per se, y facilidad en la producción de semilla (Sierra et al. 1992; Reyes 1985; Espinosa et al. 1998; Vasal et al. 1994; Vasal y Córdova 1996). Durante el ciclo primavera-verano 1998 y 1999 y otoño-invierno1997/98 y 1998/99 se condujeron siete experimentos de híbridos triples, en los que participaron como progenitores hembra las cruzas simples: H-513, CML247 x CML254 y LRB14 x D-539. Los objetivos de este trabajo fueron: 1) Conocer el rendimiento, adaptabilidad y características agronómicas de híbridos triples de maíz y de sus progenitores. 2) Determinar la aptitud combinatoria general y específica de líneas que intervienen en los híbridos triples. 3) Caracterizar a las cruzas simples que funcionan como probadores, en función de su respuesta con cada grupo de líneas.

\section{MATERIALES Y MÉTODOS}

Durante los ciclos agrícolas primavera-verano 1998 y 1999 y otoño-invierno 1997/98 y 1998/99, se condujeron siete experimentos de híbridos triples de maíz en las localidades de Cotaxtla e Isla, Veracruz, Huimanguillo, Tabasco y Apatzingán, Michoacan. Así también, durante 1999 al 2003 se establecieron parcelas de validación con los híbridos sobresalientes en los estados de Veracruz y Tabasco.

Germoplasma utilizado. Se incluyeron en la evaluación, híbridos triples de maíz, en los que intervinie- ron como progenitores hembra, las cruzas simples $\mathrm{H}$ 513, CML247 x CML254 y LRB14 x D539; y como progenitores macho fueron usados líneas sobresalientes de maíz para el trópico, entre ellas: líneas élite del Campo Cotaxtla, líneas derivadas de un compuesto de amplia base genética (CABGs), líneas recicladas de $\mathrm{H}$ 513 x VS-536 y líneas provenientes del CIMMYT.

Descripción de los experimentos. Los experimentos se establecieron bajo diseños alfa látice descrito por Barreto (1999), con dos repeticiones en parcelas de un surco de $5 \mathrm{~m}$ de largo separados a $80 \mathrm{~cm}$, depositando a la siembra dos semillas cada $20 \mathrm{~cm}$, para dejar una planta por mata, es decir, una densidad de 62.500 plantas por hectárea. En las parcelas de validación no se incluyó diseño experimental (Cuadro 1).

Cuadro 1. Descripción de los experimentos y parcelas de validación de híbridos triples de maíz. Sureste mexicano, 1998-2003.

\begin{tabular}{llrr}
\hline $\begin{array}{c}\text { Exp } \\
\text { No }\end{array}$ & \multicolumn{1}{c}{ Descripción } & $\begin{array}{c}\text { alfa- } \\
\text { látice }\end{array}$ & $\begin{array}{c}\text { No } \\
\text { trat. }\end{array}$ \\
\hline & & & \\
1 & Evaluación de híbridos trilineales COT 98A & $10 \times 9$ & 90 \\
2 & Híbridos trilineales COT98B & $12 \times 9$ & 108 \\
3 & Híbridos trilineales COT99A & $12 \times 9$ & 108 \\
4 & Ensayo uniforme de híbridos normales (4): & & \\
& Cotaxtla, Isla, Huimanguillo y Apatzingán & & \\
& 1999B & $4 \times 7$ & 28 \\
5 & $\begin{array}{l}\text { Validación de híbridos de maíz sobresalientes } \\
\end{array}$ & & \\
& Cotaxtla 1999, 2002, 2003, Balancán 2003 y & & \\
& Huimanguillo 2003 Sin diseño & 4 \\
\hline
\end{tabular}

Variables y registro de datos. Durante el desarrollo de los experimentos se registraron las siguientes variables: rendimiento de grano, altura de planta y de mazorca, días a floración masculina y femenina, aspecto de planta y de mazorca, sanidad de planta, $\%$ de acame, $\%$ de mazorcas con mala cobertura, $\%$ mazorcas podridas, $\%$ de grano y $\%$ de materia seca.

Métodos estadísticos. En los experimentos se hicieron análisis de varianza para cada una de las variables estudiadas, las variables registradas en porcentaje fueron transformadas a grados angulares (Reyes 1990). Se determinaron los valores para la Aptitud Combinatoria General (ACG) y Aptitud Combinatoria Específica (ACE) de las líneas y se hicieron análisis de regresión para los probadores. De siete experimentos establecidos, se hizo un análisis combinado y de variables de estabilidad descritas por Eberhart y Russell (1966), considerando los siguientes ambientes: 1) Cotaxtla 1998A, 2) Cotaxtla 1998B, 3) Cotaxtla 
1999A, 4) Cotaxtla 1999B, 5) Isla 1999B, 6) Huimanguillo 1999B y 7) Apatzingán 1999B.

\section{RESULTADOS Y DISCUSIÓN}

Rendimiento y características agronómicas. Del análisis combinado para rendimiento de grano en 14 híbridos triples a través de siete ambientes de evaluación, se encontró diferencia altamente significativa para las fuentes de variación Híbridos (H), Ambientes (A) y para la interacción HxA; sin embargo, fue más importante la varianza debida a los ambientes $(95,53)$. El coeficiente de variación registrado de 14,86\% indica que la conducción de los experimentos y los resultados obtenidos son confiables.

El Cuadro 2 muestra el rendimiento de híbridos triples sobresalientes a través de siete experimentos (Ambientes) donde participaron 14 genotipos comunes, en los que se aplicó el modelo de parámetros de estabilidad. Se observa que los mejores rendimientos a través de los ambientes fueron registrados para $\mathbf{H - 5 2 0}$ el cual equivale a la cruza H-513 x CABG3'-10-1-6-1-1-1, así como a las cruzas CML247 x CML254 x CABG3'-3-4-2-1-31, (LRB14 x D-539) x CML254 y al híbrido H-518 que se formó con la cruza H-513 x LT157. Así también los rendimientos promedios sugieren que los mejores ambientes se registraron en los experimentos conducidos en condiciones de riego en la localidad de Cotaxtla, por el contrario, la localidad de Huimanguillo, en condiciones de temporal registró los valores medios más bajos, debido a excesos de humedad. También se puede ver que en los híbridos experimentales sobresalientes existió una mayor frecuencia de aquellos en los que participa como progenitor hembra el H-513. En cuanto a las líneas con los probadores, podemos ver la participación mayor de las líneas derivadas de un compuesto germoplásmico de amplia base genética (CABG) y líneas provenientes del CIMMYT denominadas CMLs. Esto demuestra que existe divergencia genética entre los diferentes grupos de líneas y las cruzas simples usadas como probadores y por lo tanto hay heterosis (Reyes 1985; Gómez 1986; Vasal et al. 1992a y Vasal et al. 1992b). Esto definitivamente representa una ventaja si se considera que en los híbridos triples solo se mantienen tres progenitores y en la producción comercial se usa como progenitor hembra una cruza simple de alto rendimiento, como lo sugieren Rodríguez et al. (1997).

En relación con las características agronómicas los genotipos sobresalientes en rendimiento registran relación altura de mazorca/altura de planta cercanos al 0,50, lo que indica que son arquetipos con posibilidades en el trópico, sobretodo por su tolerancia al acame (Cuadro 3). Otras características favorables son: ciclo intermedio y buenas calificaciones de aspecto y sanidad en planta y mazorca. Se desprende de estos cuadros, que cuatro híbridos triples experimentales superan en rendimiento de grano, e igualan en las características de aspecto de planta y de mazorca al testigo comercial H-513.

Cuadro 2. Rendimiento de híbridos triples de maíz en la Región tropical. Sureste mexicano, 1998A, 1998B, 1999A y 1999B

\begin{tabular}{|c|c|c|c|c|c|c|c|c|}
\hline Trat & Genealogía & $\begin{array}{c}\text { COT } \\
99 B\end{array}$ & $\begin{array}{c}\text { ISLA } \\
\text { 99B }\end{array}$ & $\begin{array}{l}\text { HUI } \\
\text { 99B }\end{array}$ & $\begin{array}{c}\text { APA } \\
\text { 99B }\end{array}$ & $\begin{array}{c}\text { COT } \\
98 B\end{array}$ & $\begin{array}{c}\text { COT } \\
98 A\end{array}$ & $\begin{array}{c}\text { COT } \\
99 A\end{array}$ \\
\hline 4 & H-513 x CABG3'-10-1-6-1-1-1 (H-520) & 8,05 & 5,79 & 4,57 & 4,4 & 7,86 & 11,5 & 10,00 \\
\hline 8 & (CML247 x CML254) x CABG'3-3-4-2-1-3-1 & 8,56 & 4,95 & 4,87 & 5,71 & 6,66 & 10,1 & 9,21 \\
\hline 11 & (LRB14 x D-539) x CML 254 & 8,16 & 5,82 & 4,22 & 7,00 & 3,67 & 9,57 & 8,89 \\
\hline 17 & H-513 x LT157 (H-518) & 7,18 & 5,14 & 4,32 & 4,51 & 7,00 & 9,47 & 9,47 \\
\hline 19 & H-513 & 7,28 & 4,72 & 5,22 & 4,57 & 7,32 & 8,68 & 8,14 \\
\hline 12 & (LRB14 x D-539) x CABG3'-40-2-1-1-3-1 & 7,39 & 4,93 & 2,41 & 3,41 & 7,64 & 11,3 & 8,43 \\
\hline 9 & (CML 247 x CML 254) x PID-5-1-1-2-1 & 7,92 & 5,03 & 4,71 & 5,07 & 5,65 & 8,88 & 7,55 \\
\hline 1 & H-513 x CABG3'-75-2-3-1-3-1 & 6,69 & 5,1 & 4,29 & 5,55 & 6,21 & 8,13 & 8,82 \\
\hline 2 & H-513 x CABG3'-10-1-7-1-1-1 & 8,18 & 4,48 & 5,43 & 5,31 & 4,8 & 6,88 & 9,19 \\
\hline 7 & (CML247xCML254) x CABG'3-47-3-2-2-2-1 & 6,2 & 3,36 & 4,31 & 6,06 & 6,03 & 9,39 & 8,02 \\
\hline 13 & (LRB14xD-539) x CABG3'-10-1-6-1-1-1 & 7,38 & 4,47 & 4,28 & 5,14 & 2,82 & 10,1 & 8,88 \\
\hline 16 & LRB14 x D-539) x CML 264 & 6,91 & 5,25 & 4,52 & 5,83 & 6,99 & 8,88 & 4,68 \\
\hline 3 & H-513 x CABG3'-40-2-1-1-3-1 & 6,62 & 3,54 & 4,73 & 5,58 & 6,02 & 7,27 & 8,62 \\
\hline \multirow[t]{6}{*}{14} & $(\mathrm{LRB} 14 \times \mathrm{D}-539) \times$ CML 55 & 4,16 & 4,71 & 2,93 & 6,54 & 6,55 & 7,37 & 9,56 \\
\hline & (F31xF30-4-3-1 x ST549)SPLC7F254-1-1-1-3-2 & 8,44 & & & & & & \\
\hline & H-513 x SPLC7 F254-1-1-1-3-2 & 8,39 & & & & & & \\
\hline & PROM & 7,18 & 4,62 & 4,17 & 5,03 & 5,86 & 7,25 & 8,22 \\
\hline & $\mathrm{CV}(\%)$ & 12,02 & 15,78 & 18,5 & 19,3 & 20,7 & 16,7 & 11,3 \\
\hline & CME & 0,745 & 0,531 & 0,595 & 0,942 & 1,41 & 1,47 & 0,87 \\
\hline
\end{tabular}

COT = Cotaxtla, Ver,; HUI = Huimanguillo, Tab, ; APA = Apatzingán, Mich. 
Cuadro 3. Características agronómicas de híbridos de maíz. Cotaxtla, Veracruz, México. 1999B.

\begin{tabular}{|c|c|c|c|c|c|c|c|c|}
\hline $\begin{array}{c}\text { No } \\
\text { Trat }\end{array}$ & Genealogía & $\begin{array}{l}\text { Alt } \\
\text { pl }\end{array}$ & $\begin{array}{l}\text { Flor } \\
\text { Masc }\end{array}$ & $\begin{array}{c}\text { Asp } \\
\text { pl }\end{array}$ & $\begin{array}{c}\text { Asp } \\
\text { mz }\end{array}$ & $\begin{array}{c}\text { San } \\
\text { mz }\end{array}$ & $\begin{array}{c}\% \\
\text { cob }\end{array}$ & $\begin{array}{c}\% \\
\text { pod }\end{array}$ \\
\hline 1 & H-513 x CABG3'-10-1-6-1-1-1 (H-520) & 254 & 52 & 2,25 & 2 & 2 & 0,83 & 3,11 \\
\hline 2 & (CML 247 x CML 254) x CABG’3-3-4-2-1-3-1 & 262 & 52 & 2 & 1,75 & 2,25 & 1,84 & 6,5 \\
\hline 3 & (LRB14 x D-539) x CML 254 & 263 & 52 & 2,5 & 1,5 & 1,5 & 2,83 & 0 \\
\hline 4 & H-513 x LT157 (H-518) & 271 & 51 & 2,25 & 2 & 2 & 0 & 11,1 \\
\hline 5 & $\mathrm{H}-513$ & 257 & 51 & 1,75 & 1,75 & 2 & 4,06 & 1,92 \\
\hline 6 & (LRB14 x D-539) x CABG3'-40-2-1-1-3-1 & 271 & 52 & 2,75 & 2,25 & 2,75 & 0 & 3,51 \\
\hline 7 & (CML $247 \times$ CML 254) x PID-5-1-1-2-1 & 261 & 52 & 2,75 & 2 & 2 & 7,41 & 2,72 \\
\hline 8 & H-513 x CABG3'-75-2-3-1-3-1 & 279 & 53 & 2 & 2,5 & 2,75 & 2,8 & 4,56 \\
\hline 9 & H-513 x CABG3'-10-1-7-1-1-1 & 250 & 51 & 1,75 & 1,75 & 1,75 & 3,61 & 1,81 \\
\hline 10 & (CML247 x CML254) x CABG'3-47-3-2-2-2-1 & 266 & 52 & 2,25 & 2,5 & 2,5 & 13,65 & 2,72 \\
\hline 11 & (LRB14 x D-539) x CABG3'-10-1-6-1-1-1 & 265 & 51 & 1,75 & 3,25 & 3,5 & 3,42 & 8,59 \\
\hline 12 & LRB14 x D-539) x CML 264 & 244 & 53 & 2,75 & 2,5 & 2,5 & 0 & 1,82 \\
\hline 13 & H-513 x CABG3'-40-2-1-1-3-1 & 264 & 53 & 1,75 & 2,25 & 2,75 & 2,63 & 9,91 \\
\hline \multirow[t]{5}{*}{14} & (LRB14 x D-539) x CML 55 & 256 & 51 & 5 & 2,5 & 2,75 & 2,63 & 9,06 \\
\hline & F31 xF30-4-3-1 x ST549 x SPLC7F254-1-1-1-3-2 & 251 & 51 & 2,75 & 1,75 & 2,25 & 5,7 & 3,7 \\
\hline & H-513xSPLC7 F254-1-1-1-3-2 & 258 & 50 & 3 & 1,75 & 2 & 0 & 0 \\
\hline & PROMEDIO & 263,5 & 51,77 & 2,35 & 2,17 & 2,32 & 3,3 & 4,4 \\
\hline & $\mathrm{CV}(\%)$ & 4,81 & 1,36 & 21,6 & 17,6 & 13,69 & 84,71 & 85,63 \\
\hline
\end{tabular}

Adaptabilidad. Con relación a la adaptabilidad de los materiales se encontró que los híbridos superiores en rendimiento y características agronómicas fueron descritos de acuerdo con los valores de regresión y de sus desviaciones como genotipos estables, es decir, poca interacción con los cambios ambientales, lo que los define como materiales "deseables" (Cuadro 4), Así, también se encontró que los genotipos (LRB14 x
D539) x CABG3'- 40-2-1-1-3-1, y (LRB14 x D539) x CML55 tuvieron respuesta mejor en buenos ambientes pero inconsistentes.

Por lo que se refiere a los índices ambientales, en el Cuadro 5 se observa que los ambientes uno (COT98A) y tres (COT99A) registran los mejores valores, lo anterior es lógico debido a que fueron condiciones de

Cuadro 4. Parámetros de estabilidad de híbridos triples de maíz. Sureste mexicano, 1998-1999.

\begin{tabular}{rlrrrrrr}
\hline Trat & \multicolumn{1}{c}{ Genealogía } & $\begin{array}{c}\text { Rend } \\
\text { t/ha }\end{array}$ & $\begin{array}{c}\text { \% } \\
\text { Rel }\end{array}$ & Bi & S2Di & T C & Descripción \\
\hline & & & & & & & \\
1 & H-513x CABG3'-10-1-6-1-1-1 (H-520) & 7,46 & 114 & 1,43 & $-0,23$ & 2,26 & ESTABLE \\
2 & (CML 247 x CML 254) x CABG'3-3-4-2-1-3-1 & 7,15 & 109 & 1,15 & $-0,079$ & 1,77 & ESTABLE \\
3 & (LRB14 x D-539) x CML 254 & 6,76 & 103 & 1,04 & 1,08 & 0,11 & ESTABLE \\
4 & H-513 x LT157 (H-518) & 6,73 & 103 & 1,17 & $-0,6$ & 1,27 & ESTABLE \\
5 & H-513 & 6,56 & 100 & 0,85 & $-0,37$ & $-0,87$ & ESTABLE \\
6 & (LRB14 x D-539) x CABG3'-40-2-1-1-3-1 & 6,5 & 99 & 1,35 & 2,94 & 0,8 & BAI \\
7 & (CML 247 x CML 254) x PID-5-1-1-2-1 & 6,4 & 98 & 0,88 & $-0,63$ & $-0,96$ & ESTABLE \\
8 & H-513 x CABG3'-75-2-3-1-3-1 & 6,4 & 98 & 0,87 & $-0,76$ & $-1,36$ & ESTABLE \\
9 & H-513 x CABG3'-10-1-7-1-1-1 & 6,32 & 96 & 0,76 & 0,68 & $-0,84$ & ESTABLE \\
10 & (CML 247 x CML 254) x CABG'3-47-3-2-2-2-1 & 6,2 & 94 & 1,04 & $-0,17$ & 0,19 & ESTABLE \\
11 & (LRB14 x D-539) x CABG3'-10-1-6-1-1-1 & 6,15 & 94 & 1,3 & 0,94 & 0,98 & ESTABLE \\
12 & LRB14 x D-539) x CML 264 & 6,15 & 94 & 0,48 & 1,04 & $-1,64$ & ESTABLE \\
13 & H-513 x CABG3'-40-2-1-1-3-1 & 6,05 & 92 & 0,81 & $-0,17$ & $-0,98$ & ESTABLE \\
14 & (LRB14 x D-539) x CML 55 & 5,97 & 91 & 0,87 & 1,99 & $-0,34$ & BAI \\
& F31 x F30-4-3-1 x ST 549)SPLC7F254-1-1-1-3-2 & 8,44 & & & & & \\
& H-513 x SPLC7 F254-1-1-1-3-2 & 8,39 & & & & & \\
& $\quad$ PROM & 6,05 & 92 & & & & \\
& CV & 16,02 & & & & & \\
\end{tabular}

\footnotetext{
1/ Promedio de siete experimentos establecidos en Cotaxtla, Isla, Huimanguillo y Apatzingán.
}

$\mathrm{BAI}=$ respuesta mejor en buenos ambientes pero inconsistente. 
Cuadro 5. Índices ambientales registrados para los experimentos establecidos con híbridos triples en el trópico húmedo. Sureste mexicano 1998-1999.

\begin{tabular}{ccc}
\hline Ambiente & Descripción & $\begin{array}{c}\text { Índice } \\
\text { ambiental }\end{array}$ \\
\hline 1 & Cotaxtla, Ver 1998A & 2,59 \\
2 & Cotaxtla, Ver 1998B & $-0,43$ \\
3 & Cotaxtla, Ver 1999A & 2,02 \\
4 & Cotaxtla, Ver 1999B & 0,68 \\
5 & Isla, Ver 1999B & $-1,50$ \\
6 & Huimanguillo, Tab 1999B & $-2,17$ \\
7 & Apatzingán, Mich 1999B & $-1,18$ \\
\hline
\end{tabular}

riego. Por el contrario, índices ambientales negativos fueron registrados para los ambientes de Huimanguillo Tab., Isla, Ver. y Apatzingán, Mich.

Análisis de regresión para los probadores. El Cuadro 6 muestra los coeficientes de regresión de los tres probadores cruzas simples con cada grupo de líneas evaluados durante el ciclo de riego de 1998. Se encontró que los grupos de líneas CABG y Elite, registraron los mejores valores de regresión con los tres probadores; esto nos indica que existió variabilidad genética en estos dos grupos de líneas. Sin embargo, para el grupo de líneas $C A B G$ el mejor valor de regresión fue con el híbrido H-513 lo que sugiere que esta cruza permitió seleccionar a las mejores líneas. Para el grupo de líneas élites se encontró el mejor valor de regresión con el probador CML247 x CML254, esto definitivamente nos sugiere las ventajas que ofrece el aprovechamiento del germoplasma generado por el CIMMYT y el INIFAP; para los grupos de líneas CMLs y Recicladas, el probador LRB14 x D539 fue el que registró el mejor valor de regresión. Particularmente, el grupo de líneas recicladas encontró su mejor respuesta con este probador debido principalmente a que son ecológica y genéticamente más distantes, ya que LRB14 x D539 es una cruza proveniente de trópico seco en Río Bravo Tamps, e Iguala, Guerrero con grano blanco cristalino y las líneas Recicladas de H-513 x VS536 cuyo germoplasma

Cuadro 6. Coeficiente de regresión de cruzas simples con diferentes grupos de líneas. Cotaxtla, Ver., México1998A

\begin{tabular}{llcc}
\hline $\begin{array}{c}\text { Grupo } \\
\text { de líneas }\end{array}$ & H-513 & $\begin{array}{c}\text { Probadores } \\
\text { CML 247 X CML 254 }\end{array}$ & LRB-14 X D-539 \\
\hline CABG & $0,642^{*}$ & 0,567 & 0,617 \\
Elite & 0,503 & 0,807 & 0,561 \\
CML $\underline{\mathrm{s}}$ & 0,289 & 0,085 & 0,296 \\
Recicladas & 0,375 & 0,460 & 0,543 \\
\hline
\end{tabular}

es del tipo Tuxpeño con características de grano blanco dentado y adaptadas al trópico húmedo.

En cuanto al comportamiento per se de las líneas y de las cruzas simples H-513 y CML247 x CML254, en el Cuadro 7 se presenta el rendimiento de líneas que participan en los híbridos de maíz sobresalientes bajo condiciones de riego durante el ciclo otoño - invierno, en donde se observan las líneas que registraron buen rendimiento y por lo tanto facilidad para su mantenimiento y reproducción. Las cruzas registran rendimientos superiores a las 7,5 t/ha, lo que ofrece la ventaja de producción de semilla de híbridos triples al usar como progenitores hembras las cruzas simples de alto rendimiento de semilla, como lo sugieren Sierra et al. (1992), Reyes (1985), Espinosa et al. (1998), Vasal et al. (1994), Vasal y Córdova (1996).

Cuadro 7. Rendimiento de líneas progenitoras de híbridos triples sobresalientes. Cotaxtla, Ver., México. 1997-1998.

\begin{tabular}{rlrrr}
\hline Trat & \multicolumn{1}{c}{ Genealogía } & $\begin{array}{r}\text { COT } \\
\text { 1997A }\end{array}$ & $\begin{array}{r}\text { COT } \\
\mathbf{1 9 9 8 A}\end{array}$ & PROM \\
\hline & & & & \\
1 & CABG3'-10-1-6-1-1-1 & 3,66 & 3,35 & 3,51 \\
2 & CABG'3-3-4-2-1-3-1 & 3,88 & 4,62 & 4,25 \\
3 & CABG3'-40-2-1-1-3-1 & 2,93 & 3,44 & 3,19 \\
4 & PID-5-1-1-2-1 & 3,17 & 4,71 & 3,94 \\
5 & CABG3'-75-2-3-1-3-1 & 4,36 & 4,51 & 4,44 \\
6 & CABG3'-10-1-7-1-1-1 & 1,44 & 1,90 & 1,67 \\
7 & CABG'3-47-3-2-2-2-1 & 3,14 & 6,10 & 4,62 \\
8 & CML 55 & 4,07 & 3,08 & 3,58 \\
9 & F31 X F30-4-3-1 & 2,91 & 2,82 & 2,87 \\
10 & ST 549 & 2,91 & 4,20 & 3,55 \\
12 & LRB14 & 3,74 & 3,79 & 3,77 \\
13 & D-539 & 4,56 & 4,51 & 4,54 \\
14 & LT154 & 3,07 & 3,55 & 3,31 \\
15 & LT155 & 4,08 & 5,85 & 4,97 \\
16 & CML247 & 4,45 & 2,70 & 3,58 \\
17 & CML254 & 2,17 & 3,33 & 2,75 \\
18 & LE36 & 5,18 & 5,78 & 5,48 \\
19 & LE37 & 3,95 & 2,80 & 3,38 \\
20 & H-513 & 9,3 & 8,68 & 8,99 \\
21 & CML247XCML254 & 7,47 & 7,76 & 7,62 \\
& PROMEDIO & 3,16 & 3,45 & \\
& CV (\%) & 20,82 & 24,03 & \\
\hline
\end{tabular}

Validación. El Cuadro 8 resume los resultados registrados en las parcelas de validación promedio de parcelas establecidas en Cotaxtla, Veracruz durante 1999, 2002 y 2003, Balancán y Huimanguillo, Tabasco durante 2003. Se observa que el comportamiento de los híbridos H-520 (H-513 x CABG-10-1-6-1-1-1) y H-518 (H513 x LT157), fue similar en comparación con la cruza simple H-513 que participa como progenitor hembra de ambos híbridos, y $26 \%$ más rendimiento con respecto al 
Cuadro 8. Rendimiento de grano y características agronómicas de híbridos triples de maíz en parcelas de Validación. Ciclo primavera - verano en Veracruz y Tabasco ${ }^{1}$. Sureste mexicano 1999-2003.

\begin{tabular}{lccccccccc}
\hline Genealogía & $\begin{array}{c}\text { Rend } \\
\text { (t/ha) }\end{array}$ & $\begin{array}{c}\text { \% } \\
\text { Relativo }\end{array}$ & $\begin{array}{c}\text { Flor } \\
\text { masc }\end{array}$ & $\begin{array}{c}\text { Flor } \\
\text { Fem }\end{array}$ & $\begin{array}{c}\text { Altura } \\
\text { planta }\end{array}$ & $\begin{array}{c}\text { Altura } \\
\text { mazorca }\end{array}$ & $\begin{array}{c}\% \\
\text { Acame }\end{array}$ & $\begin{array}{c}\% \\
\text { cobertura }\end{array}$ & $\begin{array}{c}\text { \% mz } \\
\text { podridas }\end{array}$ \\
\hline H-520 & 7,69 & 103 & 52 & 53 & 257 & 129 & 8,2 & 4,3 & 3,2 \\
H-518 & 7,11 & 95 & 51 & 52 & 256 & 128 & 3,6 & 6,7 & 5,0 \\
H-513 & 7,50 & 100 & 51 & 52 & 237 & 121 & 6,9 & 5,0 & 3,4 \\
VS-536 & 5,80 & 77 & 52 & 53 & 266 & 141 & 15,9 & 3,8 & 4,8 \\
\hline
\end{tabular}

1 Promedio de parcelas establecidas en Cotaxtla, Ver, durante 1999, 2002 y 2003, Balancán y Huimanguillo, Tab, durante 2003.

testigo comercial VS-536. Con respecto a las características agronómicas se observan altura de planta y mazorca relativamente bajas, y buenas calificaciones de aspecto y sanidad en planta y en mazorca, tolerancia al acame y buena cobertura de la mazorca. Así también son manifiestas las ventajas en la producción de su semilla.

Se concluye de este trabajo que existen híbridos triples con buen rendimiento y estables como H-513 x CABG3'-10-1-6-1-1-1 (CML247 x CML254)xCABG3'-3-4-2-1-3-1 (LRB14 x D539) x CML254 y H-513 x LT157 que registraron de un tres al 14\% más que el testigo de cruza simple H-513. Las características agronómicas de los híbridos experimentales como son altura de planta y mazorca, aspecto de planta y mazorca y cobertura de mazorca fueron similares a los testigos $\mathrm{H}$ 513 y CML247 x CML254. Los grupos de líneas CABG y Elite registraron los mejores valores de regresión con las tres cruzas. La formación de híbridos triples representa una alternativa de aprovechamiento de la heterosis con las ventajas adicionales en la producción de semilla.

\section{LITERATURA CITADA}

BARRETO, H.J. 1999. Alpha programa para crear y analizar diseños alpha $(0,1)$ látice. In: Programa de maíz del CIMMYT manual del usuario fieldbook 5.1/7.1 y alpha México D.F. CIMMYT p. 38-48.

EBERHART S.A; RUSSELL, W.A. 1966. Stability parameters for comparing varieties. Crop Sci. 6: 36-40.

ESPINOSA, A.; ORTIZ, J.; RAMÍREZ, F. A.; GÓMEZ , N.; MARTINEZ, G. A. 1998. Estabilidad y comportamiento de líneas per se y cruzas de maíz en la producción de semilla. Agric. Téc. Méx. 24 (1) 27-36.

GÓMEZ, M.N. 1986. Aptitud combinatoria de maíces tropicales y subtropicales en la región de transición baja de Guerrero. Rev. Fitotecnia Mexicana 8: 3-19.
REYES C., P. 1985. Fitogenotecnia básica y aplicada. AGT Editor S.A. México. 460 p.

REYES. 1990. Diseño de experimentos aplicados. Ed Trillas $3^{\mathrm{a}}$ Ed. México D.F. 348 p.

RODRIGUEZ, M.F.A.; SIERRA, M.M.; CANO, R.O.; CASTAÑ́́N, N.G. 1997. Three way crosses as an alternative for producing maize in Veracruz, México. In: Coors G.M.; Pandey S. (ed.). The International Simposium about the Genetics and Exploitation of Heterosis in Crops. México city, México. p. 238.

SAGARPA (Secretaría de Agricultura, Ganadería, Desarrollo Rural, Pesca y Alimentación). 2002. Servicio de información y estadística agroalimentaria y pesquera. Subsistema de información agrícola. SAGARPA, México D.F. $\mathrm{s} / \mathrm{p}$.

SIERRA, M.; RODRÍGUEZ, F.A.; CASTILLO, R.A.; MÁRQUEZ S., F. 1992. La aplicación de los parámetros de estabilidad en el mejoramiento de maíz de la región sur de México. In: Memorias de simposium de interacción genotipo-ambiente en genotecnia vegetal. Guadalajara, Jal. p. 239-260.

VASAL, K; SRINIVASAN G. CROSSA J.; BECK D.L.1992a. Heterosis and combining ability of CIMMYT subtropical and temperate early maturity maize germplasm. Crop Sci. 32(4): 884-890.

VASAL, K; SRINIVASAN, G.; HAN, C.; GONZALEZ. F. 1992b. Heterotic patterns of eighty eigh white subtropical CIMMYT maize lines. Maydica 37: 319-327.

VASAL, K.; VERGARA, N.; MC LEAN,S. 1994. Estrategias en el desarrollo de híbridos tropicales de maíz. Agronomía Mesoamericana 5: 184-189.

VASAL, K.; CÓRDOVA O., H. 1996. Heterosis en maíz: acelerando la tecnología de híbridos de dos progenitores para el mundo en desarrollo. In: López B.A.; Rodríguez H.S.; Martínez Z.G (ed.). Memorias del Curso internacional de actualización en fitomejoramiento y agricultura sustentable. Buenavista Saltillo, Coah. p. 32-54. 